\title{
Structured Power System Model Reduction of Non-Coherent Areas
}

\author{
Christopher Sturk, Luigi Vanfretti, Yuwa Chompoobutrgool and Henrik Sandberg
}

\begin{abstract}
This paper demonstrates how structured model reduction can be used to reduce the order of power systems without the need to identify coherent groups of generators. To this end the Klein-Rogers-Kundur 2-area system is studied in detail. It is shown how different modes of the system are captured as the model order is varied, which is of interest in e.g. distributed controller design, where the objective is to damp these oscillations. The power system is divided into a study area and an external area and the proposed algorithm is used to reduce the external area to a low order linear system, while retaining the nonlinear description of the study area. It is shown that this approach permits greater deviations from the steady-state than if a reduced system that is entirely linear is used, while still yielding accurate simulation results.
\end{abstract}

Index Terms-Model reduction of power systems, internal systems, structured model reduction, dynamic equivalents

\section{INTRODUCTION}

$\mathbf{T}$ Oday's electrical power systems are immense networks of interconnected power apparatus often spanning large geographical areas, and crossing traditional national boundaries. New interconnections to neighboring networks add to the size and complexity of these systems, and this expansion trend is of particular interest in Continental Europe where additional interconnections have recently been made [1]. With this trend, new challenges for planning, operations and control of large-scale power networks will appear. This fact will make the dynamic simulation of large-scale networks [2] an even more daunting computational task for power system stability programs [3], [4], including those carrying out Dynamic Security Assessment (DSA) [5].

These complexities make the use of reduced-order dynamic equivalent models of power systems very desirable for many applications and studies, particularly for fast and cost-efficient stability assessment [6]. Research on power system model reduction is extensive, and several methodologies focusing on DSA have been implemented in software for automated model reduction [7], [6], [8]. Power system dynamic model reduction, typically known as Power System Dynamic Equivalencing [9], has the main aim of providing an equivalent system model

Invited Paper for the Panel Session: "Distributed Control - Bringing together the power and control communities", IEEE PES General Meeting 2012, San Diego, CA.

C. Sturk and H. Sandberg are with KTH Royal Institute of Technology, ACCESS Linnaeus Centre, School of Electrical Engineering, Automatic Control Lab, Stockholm, Sweden. E-mails: \{chsturk, hsan\}@kth.se. C. Sturk and H. Sandberg are supported in part by the Swedish Research Council under Grant 2007-6350 and Grant 2009-4565, and the Swedish Foundation for Strategic Research.

Y. Chompoobutrgool and L. Vanfretti are with KTH Royal Institute of Technology, School of Electrical Engineering, Electric Power Systems Division, Stockholm, Sweden. E-mails: \{yuwa, luigiv\}@kth.se. L. Vanfretti is supported by the STandUP for Energy collaboration initiative and the KTH School of Electrical Engineering. able to reproduce the aggregated steady-state [10] and dynamic characteristics of the full-order network [11], while at the same time being compatible with the available computation tools for power system analysis [8]. In this equivalent model, the study area is a portion of the network which is preserved with full detail, i.e. all the mathematical description of the power apparatus involved are untouched, while the external area, consisting of the remaining part of the network, is replaced with a simpler mathematical description, i.e. a reduced-order model.

Currently, utilities and Transmission System Operators alike make use of different techniques to compute dynamic power system equivalents. In North America, several large utilities and TSOs, such as Southern Company Services Inc. [5] and the Independent System Operator of New England (ISO-NE) [6], use those methodologies available in EPRI's DYNRED software [7]. In the case of Southern Company, an empirical method to compute power system dynamic equivalents is used in combination with DYNRED, while in ISO-NE new methods [12] implemented in DYNRED are used [6]. Regardless of the reduction technique used, they are all dependent on the use of and compliance with coherency properties.

Coherency-based power system model reduction [9], [13], [14], [15] have been well accepted by the power engineering community and automated software for their application exists [7], [6]. These methods consider two important stages, first coherency in the generators of the power system [16] is identified, and the second stage is the dynamic reduction of the system. The dynamic reduction process itself is carried out by aggregating the network [17] and aggregating the generators [18], [15]. It is even theoretically possible, in a third stage, to produce equivalent models of aggregated excitation controllers [19]. It has been recently recognized that the boundaries between the study area and the external system need to be rebuffered to properly consider system operating changes [4]. This is due to the fact that changes in operating conditions may raise variations in generator coherency behavior [20] capable of shifting the boundary of the study area to include generators that are strongly coupled to the study area. However, the generators in the new boundary may not be part of the service area of a specific utility and their dynamic models may not be available to a utility or TSO with clearly demarcated service zones. This is for example the case in Continental Europe, where CORESO [21], the regional operations coordinator for Western Europe may have limited access to dynamic models of TCS [22], a regional coordinator for the directly adjacent service area to the east of France.

Although coherency methods have been accepted as the most reliable for power system dynamic equivalencing, the major drawback is that it may not always be possible to 
reduce specific parts of the power network, the nature of coherency is to cluster generator groups which imposes the areas in which the network can be divided. To attack this limitation, some approaches that are capable of retaining a part of the network have been proposed [23], [24]. However, to address the challenges described above, and specially in the case of the different regional coordinators in Continental Europe, it would be advantageous to arbitrarily set the area corresponding to the study system without any restrictions, and without the need of satisfying coherency properties. In addition to facilitating power system security assessment [25] for the situations explained above, having such reduced models could also be beneficial in the design of power plant controllers and in the coordination of system protections for system-wide phenomena such as inter-area oscillations [1].

With the recent developments in power systems an increased interest has been seen from the automatic control community. Among other things it has been shown how model reduction algorithms popular in control can be applicable [26], [27]. These algorithms typically have a strong theoretical foundation and they are also very general in the sense that they are not targeted to a particular application. This makes them a good candidate for the reduction of power systems composed not only of synchronous machines but also of for instance renewable energy sources.

What we will use in this paper is a structured model order reduction algorithm based on balanced truncation. The idea is to reduce the external system while trying to minimize the effect it will have on the study area. This is the main objective of structured model reduction, namely to reduce models locally while ensuring a small global model error.

Model reduction where various structural constraints are taken into account ("structured model reduction") has been considered in several papers. For example, in [28] frequencyweighted model reduction problems are considered, and in [29] controller reduction is considered. More general interconnection structures have been considered in, for example, [30], [31], [32].

The structure of the paper is as follows: In Section II we formulate the problem we want to solve. Section III summarizes the theory of structured model order reduction and in Section IV it is shown how this theory can be applied to power systems. Section V introduces the Klein-Rogers-Kundur 2-area system [33] on which the merits of the proposed model reduction algorithm are evaluated.

\section{Problem Formulation}

We will assume that the power system we want to reduce can be divided into one part with system variables of interest to us, called the study area, and another part called the external area, that is only of interest in terms of its effect on the system variables in the study area (Fig. 1). This is motivated by the fact that one often only focuses on parts of the power system. Under this assumption the aim is to reduce the external area to a linear time-invariant (LTI) system of lower order, while retaining the nonlinear description of the study area. This is relevant since it allows for a physical interpretation of the reduced power system, which is helpful if one wants to simulate changes to the study area in terms of for instance transmission line failures or for the purpose of nonlinear control design. The interface between the systems is defined

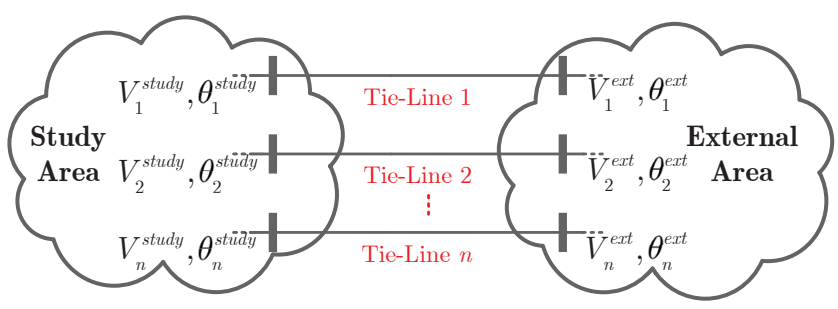

Fig. 1. The power system is divided into a study area and an external area, which is to be reduced.

by their $n$ tie-lines and buses. Given that the network with $N$ buses has an admittance matrix $Y=G+j B$, every bus satisfies the two power equations

$$
\begin{aligned}
P_{i}= & V_{i}^{2} G_{i i}+\sum_{j=1 ; j \neq i}^{N} V_{i} V_{j} B_{i j} \sin \left(\theta_{i}-\theta_{j}\right) \\
& +\sum_{j=1 ; j \neq i}^{N} V_{i} V_{j} G_{i j} \cos \left(\theta_{i}-\theta_{j}\right) \\
Q_{i}= & -V_{i}^{2} B_{i i}+\sum_{j=1 ; j \neq i}^{N} V_{i} V_{j} G_{i j} \sin \left(\theta_{i}-\theta_{j}\right) \\
& -\sum_{j=1 ; j \neq i}^{N} V_{i} V_{j} B_{i j} \cos \left(\theta_{i}-\theta_{j}\right)
\end{aligned}
$$

where $P_{i}$ and $Q_{i}$ are the injected real and reactive power respectively of bus $i$. This means that in order to have the equations (1) well-defined for the buses of the external area, it is required that it has the voltage magnitudes $V_{i}^{s t u d y}$ and phases $\theta_{i}^{\text {study }}$ that are adjacent to it as input signals. And having the external area output the voltage magnitudes $V_{i}^{\text {ext }}$ and phases $\theta_{i}^{\text {ext }}$ will ensure that the study area has all the required bus variables available to make (1) well-defined for all its buses that are adjacent to the external area. The input and output signals are analogously defined for the study area.

Given this interface the external area can be linearized around a steady-state and reduced after which it is reconnected to the study area. The objective is to do the model reduction so that the dynamics of the nonlinear study area is affected as little as possible when replacing the nonlinear description of the external area with the reduced order linear model.

\section{Model Reduction Method}

Structured model order reduction is a model reduction technique that can be applied to systems composed of subsystems that are interconnected with some network dynamics. The idea is to reduce the model order of the subsystems while retaining the interconnection structure and keeping the global model error small. The general system (Fig. 2) to which structured model reduction can be applied is composed of $q$ subsystems 
described by the transfer function matrix

$$
G(s)=\left[\begin{array}{ccc}
G_{1}(s) & & 0 \\
& \ddots & \\
0 & & G_{q}(s)
\end{array}\right]=:\left[\begin{array}{c|c}
A_{G} & B_{G} \\
\hline C_{G} & D_{G}
\end{array}\right]
$$

with transfer functions $G_{k}(s) \in \mathbb{C}^{p_{k} \times m_{k}}$ and an interconnecting network

$$
N(s)=\left[\begin{array}{c|c}
E(s) & F(s) \\
\hline H(s) & K(s)
\end{array}\right]=:\left[\begin{array}{c|cc}
A_{N} & B_{N, 1} & B_{N, 2} \\
\hline C_{N, 1} & D_{E} & D_{F} \\
C_{N, 2} & D_{H} & D_{K}
\end{array}\right] .
$$

We want to find a reduced order system that approximates the

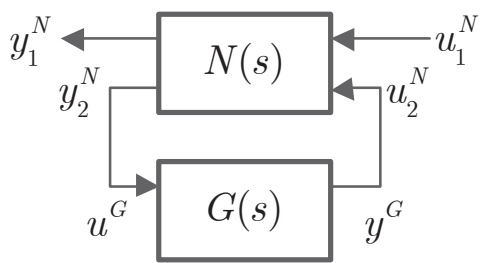

Fig. 2. The interconnected system. $G$ is the system that should be reduced and $N$ is the interconnecting network. $w$ and $z$ are external input and output respectively. $u$ and $y$ are the input and output to the system that should be reduced.

mapping from $u_{1}^{N}$ to $y_{1}^{N}$ defined by the lower linear fractional transformation

$$
\begin{aligned}
& \mathcal{F}_{l}(N, G):=E(s)+F(s)(I-G(s) K(s))-1 G(s) H(s)= \\
& {\left[\begin{array}{cc|c}
A_{N}+B_{N, 2} L D_{G} C_{N, 2} & B_{N, 2} L C_{G} & B_{N, 1}+B_{N, 2} L D_{G} D_{H} \\
B_{G} M C_{N, 2} & A_{G}+B_{G} M D_{K} C_{G} & B_{G} M D_{H} \\
\hline C_{N, 1}+D_{F} D_{G} M C_{N, 2} & D_{F} L C_{G} & D_{E}+D_{F} D_{G} M D_{H}
\end{array}\right]} \\
& =:\left[\begin{array}{c|c}
A & B \\
\hline C & D
\end{array}\right], \quad L:=\left(I-D_{G} D_{K}\right)^{-1}, \quad M:=\left(I-D_{K} D_{G}\right)^{-1},
\end{aligned}
$$

with the constraint that the interconnecting network $N$ is retained. So with $\|\cdot\|_{\infty}$ being defined as the induced $\mathcal{L} 2$ norm [34], the objective is to find the reduced order system $\hat{G}$ such that

$$
\left\|\mathcal{F}_{l}(N, G)-\mathcal{F}_{l}(N, \hat{G})\right\|_{\infty}
$$

is made as small as possible and

$$
\hat{G} \in\left\{F(s): F(s)=\operatorname{diag}\left\{F_{1}(s), \ldots, F_{q}(s)\right\}\right\}
$$

where $F_{k}(s) \in \mathbb{C}^{p_{k} \times m_{k}}, k=1, \ldots, q$. This can be contrasted with [35], where the model reduction of the external area $G$ is made independently of the study area $N$. The reason for incorporating $N$ in the model reduction is that it amplifies the frequencies of $G$ with different gains, and this will affect which modes of $G$ that are the most important to retain a good model of.

Finding the optimal minimum to (5) is very difficult, since it is a nonconvex optimization problem. We will therefore have to resort to suboptimal methods, which yield solutions satisfying the constraints while trying to minimize the norm of the model error. The model order reduction algorithm used in this paper is based on the idea of balanced truncation, see for example [28] and [36]. To enforce the structural constraints we use a generalization of balanced truncation as described in [37], [38], [31], [32]. The notation used here closely follows the one used in [31] and [32].
It uses the reachability and observability Gramians $P$ and $Q$ given by the Lyapunov equations

$$
A P+P A^{T}+B B^{T}=0, \quad A^{T} Q+Q A+C^{T} C=0,
$$

with the matrices $A, B, C$ defined by (4). It is helpful to use a partition with the blocks $Q_{N}, P_{N}$ for the interconnecting network that is not reduced and with the blocks $Q_{k}, P_{k}$ for subsystem $k$ that should be reduced separately, but in a way so that the closed-loop dynamics is retained.

$$
\begin{gathered}
Q=\left[\begin{array}{cc}
Q_{N} & Q_{N G} \\
Q_{N G}^{T} & Q_{G}
\end{array}\right], Q_{G}=\left[\begin{array}{ccc}
Q_{1} & \ldots & Q_{1 q} \\
\vdots & \ddots & \vdots \\
Q_{1 q}^{T} & \cdots & Q_{q}
\end{array}\right] \\
P=\left[\begin{array}{cc}
P_{N} & P_{N G} \\
P_{N G}^{T} & P_{G}
\end{array}\right], P_{G}=\left[\begin{array}{ccc}
P_{1} & \ldots & P_{1 q} \\
\vdots & \ddots & \vdots \\
P_{1 q}^{T} & \cdots & P_{q}
\end{array}\right]
\end{gathered}
$$

The method balances the subsystems $G_{k}(s)$ by the coordinate transformation $x_{k}=T_{k} \bar{x}_{k}$ that makes the transformed Gramians $\bar{Q}_{k}=T_{k}^{T} Q_{k} T_{k}$ and $\bar{P}_{k}=T_{k}^{-1} P_{k} T_{k}^{-T}$ subsystem balanced, which means that

$$
\begin{aligned}
& \bar{P}_{k}=\bar{Q}_{k}=\Sigma_{k}=\operatorname{diag}\left\{\sigma_{k, 1}, \ldots, \sigma_{k, n_{k}}\right\}, \\
& \sigma_{k, 1} \geq \ldots \geq \sigma_{k, n_{k}}, \\
& \sigma_{k, j}=\sqrt{\lambda_{j}\left(P_{k} Q_{k}\right)}=\sqrt{\lambda_{j}\left(\bar{P}_{k} \bar{Q}_{k}\right)} .
\end{aligned}
$$

Thus if the original state vector has the structure,

$$
x=\left[\begin{array}{llll}
x_{N}^{T} & x_{1}^{T} & \ldots & x_{q}^{T}
\end{array}\right]^{T},
$$

then the transformed system will have the states $\bar{x}$ defined by

$$
T \bar{x}=x,
$$

where

$$
T=\operatorname{diag}\left(T_{N}, T_{1}, \ldots, T_{q}\right), T_{N} \in \mathbb{R}^{n_{N} \times n_{N}}, T_{k} \in \mathbb{R}^{n_{k} \times n_{k}},
$$

and $n_{N}$ and $n_{k}$ are the order of system $N$ and $G_{k}$ respectively.

Having made a coordinate transformation, either truncation or singular perturbation is used to calculate the reduced order systems $\hat{G}_{i}$ of the subsystems in $G$. To this end the structured Hankel singular values can guide the choice of which states to retain as explained below.

The strength of the structured model reduction algorithm is that the block-diagonal elements of the Gramians defined by (6) tell us how reachable and observable the states of the subsystems are when we control the global input signal $u_{1}^{N}$ and observe the global output signal $y_{1}^{N}$ (Fig. 2).

\section{Structured Model Reduction of Power SYSTEMS}

The structured model reduction algorithm accounted for in Section III is based on the notion of dividing the system into the subsystems $N$ and $G$. This makes it suitable for application to power systems with one study area, which we want to retain a nonlinear description of and one external area which we want to reduce. We will henceforth assume that the system $G$ only consists of one external area that we want 
to reduce, i.e. with the notation introduced in Section III, $q=1$. Although we make this assumption, there is nothing preventing $G$ from being composed of more than one external area. We now propose the following four-step algorithm:

\section{Defining the model}

A general power system will be described by differential algebraic equations (DAE) of the form

$$
\begin{aligned}
& \dot{x}=f\left(x, x_{\mathrm{alg}}, u\right) \\
& 0=g\left(x, x_{\mathrm{alg}}, u\right) .
\end{aligned}
$$

The states $x$ will be describing the generators, controllers, etc., whereas the algebraic variables $x_{\text {alg }}$ will be the voltages and phases of the buses as well as algebraic variables describing the generators, controllers, etc. The signal $u$ will be an exogenous input to the power system. It could for instance describe time-varying loads or a reference signal to a controller. Divide this system into a study area, denoted $N$ and an external area denoted $G$. A general system with this structure can be described by the DAEs

$$
\begin{aligned}
& \dot{x}^{G}=f^{G}\left(x^{G}, x_{\mathrm{alg}}^{G}, u^{G}\right) \\
& 0=g^{G}\left(x^{G}, x_{\mathrm{alg}}^{G}, u^{G}\right)
\end{aligned}
$$

and

$$
\begin{aligned}
& \dot{x}^{N}=f^{N}\left(x^{N}, x_{\text {alg }}^{N}, u_{1}^{N}, u_{2}^{N}\right) \\
& 0=g^{N}\left(x^{N}, x_{\text {alg }}^{N}, u_{1}^{N}, u_{2}^{N}\right)
\end{aligned}
$$

The variables $u^{G}$ and $u_{2}^{N}$ are the voltage magnitudes and phases of the buses at the tie-line as described in Section II and $u_{1}^{N}$ is the same exogenous input as in (11), (Fig. 2).

\section{Linearizing}

In order to apply the structured model reduction algorithm described in Section III it is first necessary to linearize both the study area and the external area. By solving the power flow problem, the steady-state of the power system is acquired around which the linearization is done. The linearization of $(12,13)$ will take the form

$$
\begin{aligned}
\left(\begin{array}{l}
\dot{x}^{G} \\
0
\end{array}\right)=\left(\begin{array}{ll}
A_{11}^{G} & A_{12}^{G} \\
A_{21}^{G} & A_{22}^{G}
\end{array}\right)\left(\begin{array}{l}
x^{G} \\
x_{\mathrm{alg}}^{G}
\end{array}\right)+\left(\begin{array}{l}
B_{1}^{G} \\
B_{2}^{G}
\end{array}\right) u^{G} \\
\left(\begin{array}{l}
\dot{x}^{N} \\
0
\end{array}\right)=\left(\begin{array}{ll}
A_{11}^{N} & A_{12}^{N} \\
A_{21}^{N} & A_{22}^{N}
\end{array}\right)\left(\begin{array}{l}
x^{N} \\
x_{\mathrm{alg}}^{N}
\end{array}\right) \\
+\left(\begin{array}{ll}
B_{11}^{N} & B_{12}^{N} \\
B_{21}^{N} & B_{22}^{N}
\end{array}\right)\left(\begin{array}{l}
u_{1}^{N} \\
u_{2}^{N}
\end{array}\right) .
\end{aligned}
$$

What makes these systems easy to work with is that the algebraic variables $x_{G}^{a l g}$ and $x_{N}^{a l g}$ can be solved for

$$
\begin{gathered}
x_{\mathrm{alg}}^{G}=-A_{22}^{G^{-1}}\left(A_{21}^{G} x^{G}+B_{2}^{G} u^{G}\right) \\
x_{\mathrm{alg}}^{N}=-A_{22}^{N^{-1}}\left(A_{21}^{N} x^{N}+B_{21}^{N} u_{1}^{N}+B_{22}^{N} u_{2}^{N}\right) .
\end{gathered}
$$

If the matrices $M^{G}$ and $M^{N}$ select which algebraic variables the two subsystems output, i.e. the tie-line voltage magnitudes and phases, the DAEs can be recast into the following ordinary differential equations

$\dot{x}^{G}=\left(A_{11}^{G}-A_{12}^{G} A_{22}^{G^{-1}} A_{21}^{G}\right) x^{G}+\left(B_{1}^{G}-A_{12}^{G} A_{22}^{G^{-1}} B_{2}^{G}\right) u^{G}$

$y^{G}=M^{G}\left(-A_{22}^{G^{-1}}\left(A_{21}^{G} x^{G}+B_{2}^{G} u^{G}\right)\right)$ and

$$
\begin{aligned}
& \dot{x}^{N}=\left(A_{11}^{N}-A_{12}^{N} A_{22}^{N^{-1}} A_{21}^{N}\right) x^{N} \\
& +\left[\begin{array}{lll}
\left(B_{11}^{N}-A_{12}^{N} A_{22}^{N^{-1}} B_{21}\right) & \left(B_{12}^{N}-A_{12}^{N} A_{22}^{N^{-1}} B_{22}\right)
\end{array}\right]\left(\begin{array}{c}
u_{1}^{N} \\
u_{2}^{N}
\end{array}\right) \\
& \left(\begin{array}{l}
y_{1}^{N} \\
y_{2}^{N}
\end{array}\right)=\left(\begin{array}{l}
0 \\
M^{N}
\end{array}\right)\left(-A_{22}^{N^{-1}}\left(A_{21}^{N} x^{N}+B_{21}^{N} u_{1}^{N}+B_{22}^{N} u_{2}^{N}\right)\right) \\
& +\left(\begin{array}{ll}
0 & I \\
0 & 0
\end{array}\right)\left(\begin{array}{l}
u_{1}^{N} \\
u_{2}^{N}
\end{array}\right)
\end{aligned}
$$

We can note that the system $N$ has one input signal $u_{1}^{N}$ and one output signal $y_{1}^{N}$ apart from the input-output pair that defines its interconnection with the external area $G$. These are the exogenous inputs and the global outputs and they will be elaborated upon in Subsection IV-A.

\section{Model reduction}

With $G$ and $N$ on the form (14) and (15) the state space equations for the interconnected system is readily found by using (4) for which the reachability and observability Gramians can be calculated with (6). Selecting the submatrices $P_{G}$ and $Q_{G}$ from the matrices (8) and (7) a change of coordinates for the system $G$ can be found and guided by the structured Hankel singular values (9) the model order can be selected.

\section{Nonlinear model}

With the system $G$ being reduced to

$$
\hat{G}(s)=\left[\begin{array}{c|c}
A_{\hat{G}} & B_{\hat{G}} \\
\hline C_{\hat{G}} & D_{\hat{G}}
\end{array}\right]
$$

it can be reconnected to the nonlinear description of the study area yielding the reduced interconnected system

$$
\begin{aligned}
& \dot{x}^{\hat{G}}=A_{\hat{G}} x^{\hat{G}}+B_{\hat{G}} u^{\hat{G}} \\
& u_{2}^{N}=y_{\hat{G}}=C_{\hat{G}} x^{\hat{G}}+D_{\hat{G}} u^{\hat{G}} \\
& \dot{x}^{N}=f^{N}\left(x^{N}, x_{\mathrm{alg}}^{N}, u_{1}^{N}, u_{2}^{N}\right) \\
& 0=g^{N}\left(x^{N}, x_{\mathrm{alg}}^{N}, u_{1}^{N}, u_{2}^{N}\right) \\
& u^{\hat{G}}=y_{2}^{N}=M_{N} x_{\mathrm{alg}}^{N} .
\end{aligned}
$$

\section{A. Algorithm preferences}

Having linearized the system, we want to find the reduced system $\hat{G}$ that makes (5) as small as possible. The outcome of the model reduction will of course be dependent on the choice of exogenous input signals $u_{1}^{N}$ and output signals $y_{1}^{N}$. In the Klein-Rogers-Kundur 2-area system that will be used to demonstrate the algorithm, the input signal $u_{1}^{N}$ was chosen as the voltage reference $V_{r e f, 1}$ of the AVR of generator 1 (Fig. 3 ). This choice is of interest if one wants to design a power system stabilizer (PSS), since such a controller is dependent on a good model with $V_{\text {ref,1 }}$ as input and that is exactly what the reduced model will provide.

A natural choice for the output variables $y_{1}^{N}$ is to choose them as the voltage magnitudes and phases of the buses of the external area at the tie-lines. Alternatively one may use the voltage magnitude as before together with the phase difference of the buses connected by tie-lines as outputs, i.e. $\theta_{6}-\theta_{5}$ in 
this case, and this is what is done here. By assuring that the magnitude of the voltage and the phase difference of the buses connected by tie-lines are accurately modeled, it immediately follows that the impact of the external area on the study area is properly modeled, since these are the variables that define the magnitude of the injected power into the study area. And since the study area is not reduced, its dynamics will be the same as in the full system as long as the injected power is accurately modeled.

\section{B. Required assumptions}

The structured model reduction algorithm, which is based on balanced truncation, requires that the interconnected system is asymptotically stable, since the reachability and observability Gramians satisfying (6) will not be well-defined otherwise. A power system with only synchronous generators and loads will however always have an eigenvalue at the origin as long as no reference for the voltage phase angles is chosen. What we will do in this paper is to introduce a very small artificial feedback from the terminal voltage phase angle at generator 3 in the external area. This feedback term will be used to modify the voltage reference of the AVR so that it becomes $V_{\text {ref }, 3}=\hat{V}_{\text {ref }, 3}+k \theta_{3}$ (Figure 3), where $\hat{V}_{\text {ref }, 3}$ is the original reference value and $k$ is a very small number selected by the user. The effect of this, is that the eigenvalue at the origin will be pushed slightly into the left half-plane and the distance it is moved will be a function of $k$, so by choosing $k$ very small, the eigenvalue will be very close to the origin. This will render the system asymptotically stable, thus allowing for the computation of the reachability and observability Gramians.

The reason that the power system has an eigenvalue in the origin in the first place, is that there is no absolute reference phase, since all that matters are the phase differences. This means that the power system would not be affected if all the phases were shifted by an offset. However, by introducing this artificial feedback term, a shift in all the phases would change the dynamics, and this explains why the eigenvalue at the origin will move. This also means that the other eigenvalues of the system will move slightly, but by choosing $k$ very small, the change will be insignificant. This is important since it means that we can work with the perturbed system to compute the reduced system. The only thing that one needs to be aware of is that the phase drift of the system at the presence of some perturbation will not be the same for the reduced system as for the full system. But again, it is not the absolute phase that is of importance, but the difference in phases between the buses, because it is the difference that defines the power flow in the transmission lines.

\section{Algorithm Demonstration}

To compute the reduced order power system we have used the Power System Analysis Toolbox (PSAT) [39]. Its Simulink interface can be used to draw the two subsystems $N$ and $G$. After using the built-in method to solve the power flow problem, PSAT can return the two linearized subsystems.

Then to simulate the reduced order system at the presence of some perturbation, MATLAB's ode15s solver for stiff DAEs of index 1 was used. This particular solver was chosen because power systems with high-order generators and Automatic Voltage Regulators (AVR) are known to have both really fast and slow dynamics, i.e. they are stiff systems [9]. These simulations were compared with simulations in PSAT of the full system. The algorithm used in PSAT is based on an implicit trapezoidal rule method.

\section{A. Model}

The model we will study is the Klein-Rogers-Kundur 2area system [33] (Figure 3) ${ }^{1}$. It will be shown how different features of it can be captured as the model order of the reduced model is varied. In particular we are interested in the three main modes of the system, which are the inter-area mode with frequency $0.48 \mathrm{~Hz}$ and damping 0.032 that causes machine 1 and 2 to swing against machine 3 and 4 , and the two intra-area modes. The first intra-area mode causes machine 1 to swing against machine 2 with a frequency of $0.95 \mathrm{~Hz}$ and damping 0.089 , and the second mode makes machine 3 swing against machine 4 with a frequency of $0.98 \mathrm{~Hz}$ and damping 0.089 . Since the inter-area mode is the mode with the least damping, we can expect that it will be important to get it right in order to achieve a small model error. We have selected machine 1

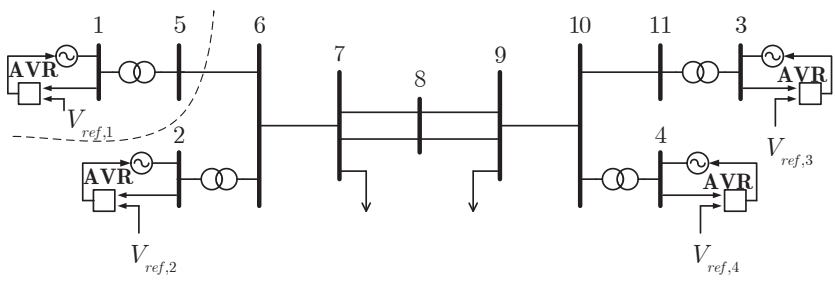

Fig. 3. The Klein-Rogers-Kundur 2-area system divided into a study area including generator 1 and an external area, which is to be reduced.

together with bus 1 and 5 to be the study area $N$. The study area is connected to the external area $G$, which is the rest of the network, via the tie-line between bus 5 and 6 .

The generators are all modeled as sixth order synchronous machines and they are controlled by fourth order AVRs of the standard IEEE model 1.

\section{B. Results}

To validate the reduced model we will look at how well it can retain the modes of key importance. This can be done by looking at the eigenvalues of the reduced model at the steady-state operating point and comparing them with those of the full system. The importance of preserving the location of the eigenvalues corresponding to the inter-area oscillation as well as the intra-area oscillations can then be demonstrated by simulating the system when it is perturbed. By increasing the model order of the reduced system, more modes are captured and higher model fidelity is attained.

\footnotetext{
${ }^{1}$ The parameters are the same as in [33], with the only difference that a damping $D=1$ has been added to all generators and that the saturation limit of the regulator voltage of the AVRs has been changed from \pm 5 to \pm 2.5 .
} 
1) Preservation of key modes: Studying the eigenvalues of a system is relevant since it gives information about the modes of the system in terms of their frequency and damping. For this reason it also becomes interesting to see which eigenvalues of the reduced system that are preserved for different model orders and how well their frequency and damping agree with those of the full system. Since the main modes of interest for the Klein-Rogers-Kundur 2 area system are the interarea mode and the two intra-area modes we will restrict our attention to those. In Table V-B1 it is shown how well these modes are preserved for different model orders. Note that the model order refers to the order of the reduced system $G$. We can see that it is sufficient to model the external area as a fourth order system if we are primarily interested in capturing the inter-area mode. The frequency and the damping are almost identical to the values of the full system. However the intraarea mode between generator 1 and 2 is not modeled very accurately, and the intra-area mode between the generators 3 and 4 does not even exist for this model order. This is no surprise though, as the external area is only modeled as a fourth order system. With the inter-area mode being the most dominant and long-lived mode, it is reasonable that a good model emphasizes the accuracy of it over the other modes.

As the model order is increased from 4 to 7 , it can be seen from the table that better precision is achieved for the intra-area mode of generator 1 and 2, but that the other intraarea mode is still missing. As it turns out, the model order has to be increased to 12 , to capture it. This can most likely be attributed to the choice of exogenous input signals to the power system. Since the only input signal, $V_{\text {ref, }, 1}$, primarily excites generators 1 and 2, the model reduction algorithm will automatically assign greater importance to capturing that intraarea mode. By choosing different input signals or by adding for instance a voltage reference to one of the generators 3 or 4 as an additional exogenous input signal, more importance would be assigned to capturing the intra-area mode of generator 3 and 4 .

2) Time-simulations: We now want to see if the preservation of the key eigenvalues is reflected in the simulations of the different models. To excite the system the input signal $V_{r e f, 1}$ will be used and to validate the reduced model, the variables $V_{6}$ and $\theta_{6}-\theta_{5}$ will be chosen. This is a natural choice, since these variables define the injected power into the study area from the external area and if that injected power is well modeled, the study area will behave well as a result, since the nonlinear description is used in the reduced model.

Before exciting the system with $V_{r e f, 1}$ however, we will begin by considering a $1 \%$ perturbation of the synchronous machine internal angle $\delta$ of generator 1 . The transients that this gives rise to are shown in Figure 4, where $\theta_{6}-\theta_{5}$ is being plotted. Note that the model order refers to the order of the reduced system $G$. In blue, we have the simulation of the full system. We see that there is an initial excitation of the intraarea mode between generator 1 and 2, which quite rapidly fades. Much longer lasting is the inter-area mode. Comparing this transient with those of the reduced models, it can be seen that the fourth order model in black does not quite capture the intra-area mode, but once that mode has died out, leaving only the inter-area mode, the match with the full system is almost perfect. Looking at what eigenvalues it preserves (Table V-B1), this seems reasonable. The eigenvalues of the intra-area modes are not as well modeled as the eigenvalue describing interarea mode and this is reflected in the time-simulations. From

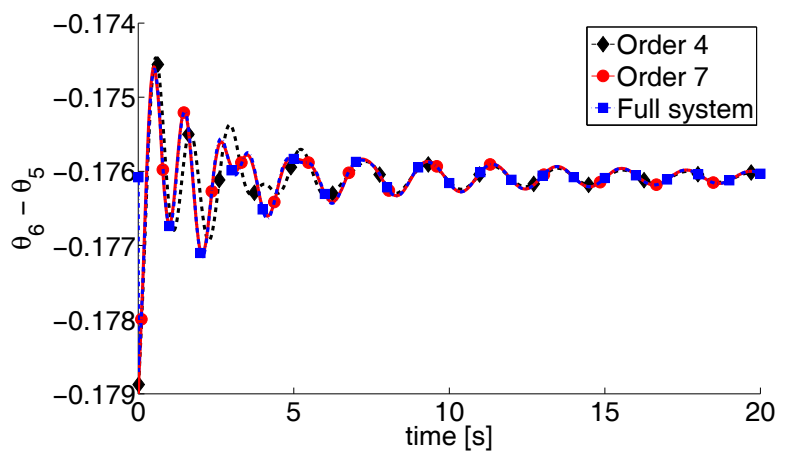

Fig. 4. The system is perturbed by offsetting the initial value of the synchronous machine internal angle $\delta$ of generator 1 by $1 \%$.

the table it can be noted that the accuracy of the intra-area modes increases with the number of retained states, and thus it should be expected that the time-simulations show a similar improvement as the number of states is increased. Looking at the transients for the system of order 7, this is indeed so.

Another natural simulation to run is one where $V_{r e f, 1}$ of generator 1 perturbed from its steady-state value. It will here be modeled as a step signal and we will see what happens as the amplitude of the step is increased. To begin with, consider an amplitude of $1 \%$ of the steady-state value. The results are shown in Figure 5, where $V_{6}$ has been plotted as a function of time. The fourth order system again captures the inter-area oscillation although there is an offset. It is also seen that a seventh order system is sufficient to get an almost perfect fit.

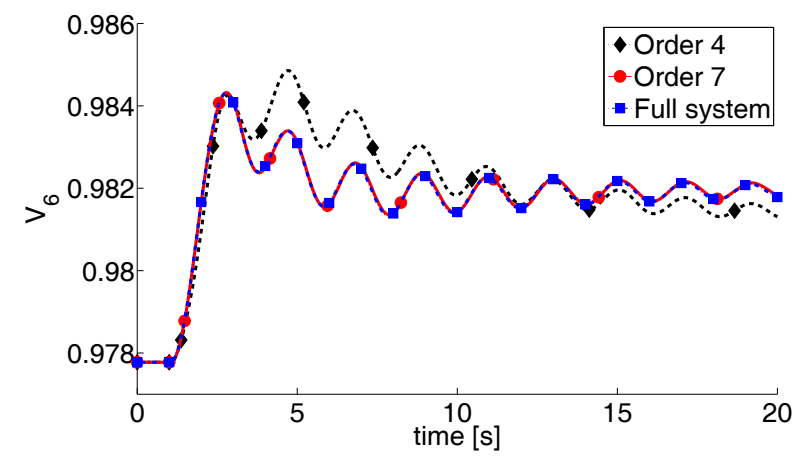

Fig. 5. A step in $V_{r e f, 1}$ with amplitude $1 \%$ is applied.

3) Moving away from small-signal analysis: So far we have only looked at small deviations from the steady-state of the system. It is well-known that linear models work well for this kind of small-signal analysis, but as the trajectory moves further away from the equilibrium, the nonlinearities will start to kick in. By retaining a nonlinear description of the study area, the idea is that it should be possible to get better accuracy of the system, than if it was modeled as an entirely linear system. To demonstrate this, first consider a small excitation 
TABLE I

Eigenvalues of a Selection of Reduced Order Models Compared to the Full-Order Linear Model

\begin{tabular}{|c|c|c|c|c|c|c|c|c|c|}
\hline \multirow{2}{*}{$\begin{array}{c}\text { Model Order } \\
\text { No. }\end{array}$} & \multicolumn{2}{|c|}{ Inter-Area Mode $\left(\lambda_{1}\right)$} & \multicolumn{2}{c|}{ Local Mode $1\left(\lambda_{2}\right)$} & \multicolumn{3}{c|}{ Local Mode $2\left(\lambda_{3}\right)$} \\
\cline { 2 - 10 } & $\lambda_{1}$ & $f_{1}(\mathrm{~Hz})$ & $\zeta_{1}(\%)$ & $\lambda_{2}$ & $f_{2}(\mathrm{~Hz})$ & $\zeta_{2}(\%)$ & $\lambda_{3}$ & $f_{3}(\mathrm{~Hz})$ & $\zeta_{3}(\%)$ \\
\hline 4 & $-0.103 \pm j 3.02$ & 0.481 & 3.41 & $-0.564 \pm j 5.30$ & 0.844 & 10.6 & - & - & - \\
\hline 7 & $-0.0977 \pm j 3.03$ & 0.482 & 3.22 & $-0.599 \pm j 5.97$ & 0.950 & 9.98 & - & - & - \\
\hline 12 & $-0.0970 \pm j 3.03$ & 0.482 & 3.20 & $-0.537 \pm j 5.99$ & 0.953 & 8.93 & $-0.786 \pm j 5.63$ & 0.896 & 13.8 \\
\hline full system & $-0.0970 \pm j 3.03$ & 0.482 & 3.20 & $-0.538 \pm j 6.00$ & 0.955 & 8.93 & $-0.550 \pm j 6.17$ & 0.982 & 8.88 \\
\hline
\end{tabular}

of $V_{r e f, 1}$ with an amplitude of $1 \%$ of the steady-state value. This time we will be looking at a $12 t h$ order system $G$ and we will see what difference it makes if it is connected to a linear model as opposed to a nonlinear model of the study area. In Figure 6, it can be seen that it has no major importance whether the linear or nonlinear system $G$ is used, which is to be expected, since the deviation from the equilibrium is so small. If the amplitude of the input signal $V_{r e f, 1}$ on the other

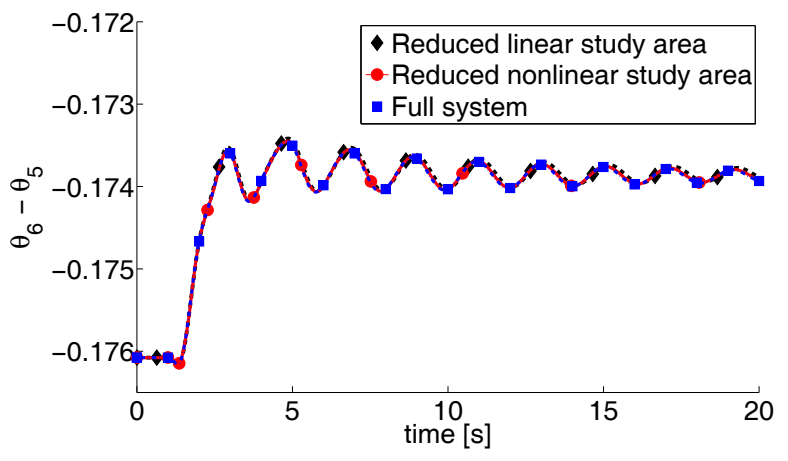

Fig. 6. A step in $V_{r e f, 1}$ with amplitude $1 \%$ is applied. The model order of the external area $G$ is 12 . A comparison is made between a linear and a nonlinear representation of the study area.

hand is set to $20 \%$ of its steady-state value, the outcome is quite different (Figure 7). With an entirely linear system, all that happens is that the output is scaled by a factor 20. But the full system is nonlinear and as such its dynamics can look different at different points in the state-space, which can be seen to be the case from this time-simulation. The transient of the reduced nonlinear model does not match perfectly with the transient of the full system, but it can be seen that it captures most of the nonlinear dynamics which is lost in the linear simulation. In fact, the nonlinearity that contributes the most to the results seen in Figure 7 is the saturation of the field voltage of generator 1. Figure 8 shows this very clearly. Since the study area of the reduced nonlinear model contains an exact model of generator 1, its transient look almost identical to that of the full nonlinear model. For an entirely linear model however it is impossible to capture the effects of saturation and this is what is seen from the figure.

\section{CONCLUSION AND DISCUSSION}

This paper has demonstrated the merits of applying structured model order reduction to power systems by analyzing the Klein-Rogers-Kundur 2 area system. It was seen that this system could be substantially reduced without losing much accuracy. With this we have shown the potential of

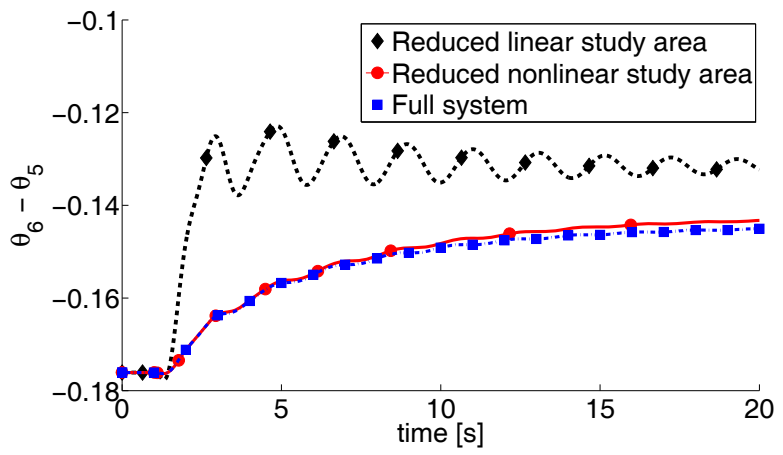

Fig. 7. A step in $V_{\text {ref }}$ with amplitude $20 \%$ is applied. The model order of the external area $G$ is 12 . A comparison is made between a linear and a nonlinear representation of the study area.

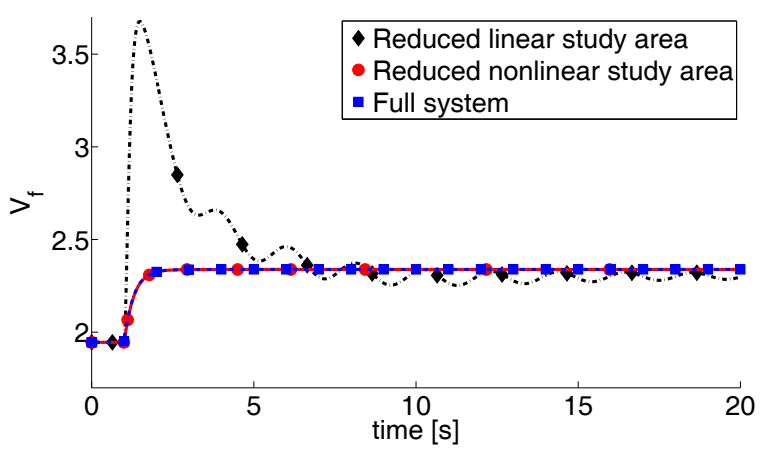

Fig. 8. A step in $V_{\text {ref }}$ with amplitude $20 \%$ is applied. The model order of the external area $G$ is 12 . A comparison is made between a linear and a nonlinear representation of the study area.

the algorithm, but future work should include the study of large-scale systems. Since the proposed algorithm is based on balanced truncation, the computational complexity of the two algorithms will also be comparable. Balanced truncation has typically been considered suitable for up to medium-sized systems with a few thousand states [40]. However there has been work on approximative balanced reduction algorithms, which extends its applicability to large-scale systems [40].

The proposed algorithm bears similarities to the inertial and slow coherency approach [15], in the sense that the external system is reduced to a linearized network. However this algorithm has the advantage that it is not dependent on coherency properties of the external area. This was seen from the model reduction of the Klein-Rogers-Kundur 2 area system, where the external area was chosen to be non-coherent.

It could be argued that the current approach has one practical limitation, which is that there is no mapping to a 
"physical component" of the linear external area as done in [15]. This is a problem because today's power system analysis tools are not written to simultaneously simulate detailed DAE descriptions of the study area and linear models of the external area. However, this issue can easily be dealt with, as shown in this paper, by adding a linear component to the simulation software.

An advantage of the algorithm, although not illustrated in this paper, is that it easily extends to systems with several study areas, which is relevant for power system regional coordinators in Europe, such as CORESO, that have study areas whose boundaries do not overlap.

\section{REFERENCES}

[1] W. Sattinger, "Application of PMU measurements in Europe TSO approach and experience," in IEEE Trondheim PowerTech 2011, June 2011, pp. $1-4$.

[2] M. Butts and H. Smith, "Dynamic oscillations predicted by computer studies," IEEE Computer Applications in Power, vol. 4, no. 1, pp. 47 -51 , jan 1991

[3] J. Vernotte, P. Panciatici, B. Meyer, J. Antoine, J. Deuse, and M. Stubbe, "High fidelity simulation of power system dynamics," IEEE Computer Applications in Power, vol. 8, no. 1, pp. 37 -41, jan 1995.

[4] S. Lee and F. Schweppe, "Distance Measures and Coherency Recognition for Transient Stability Equivalents," IEEE Transactions on Power Apparatus and Systems, vol. PAS-92, no. 5, pp. 1550 -1557, sept. 1973.

[5] J. Viikinsalo, A. Martin, K. Morison, L. Wang, and F. Howell, "Transient Security Assessment in Real-time at Southern Company," in IEEE PES Power Systems Conference and Exposition (PSCE '06), 29 2006-nov. 1 2006, pp. $13-17$.

[6] F. Ma, X. Luo, and V. Vittal, "Application of dynamic equivalencing in large-scale power systems," in IEEE Power and Energy Society General Meeting, july 2011, pp. $1-10$.

[7] R. Podmore, "Development of dynamic equivalents for transient stability studies," EPRI, Report EPRI Project 763, May 1997.

[8] F. Milano and S. K., "Dynamic REI equivalents for short circuit and transient stability analysis," Electric Power Systems Research, vol. 79, pp. 878-887, 2009.

[9] J. H. Chow, G. Peponides, P. V. Kokotovic, B. Avramovic, and J. R. Winkelman, Time-Scale Modeling of Dynamic Networks with Applications to Power System, J. H. Chow, Ed. New York: Springer-Verlag, 1982.

[10] M. Oatts, S. Erwin, and J. Hart, "Application of the REI equivalent for operations planning analysis of interchange schedules," in Power Industry Computer Application Conference, 1989. PICA '89, Conference Papers, may 1989, pp. $424-430$.

[11] G. Rogers, Power System Oscillations. Norwell, MA: Kluwer Academic Publishers, 1999.

[12] F. Ma and V. Vittal, "Right-Sized Power System Dynamic Equivalents for Power System Operation," IEEE Transactions on Power Systems, vol. 26, no. 4, pp. $1998-2005$, nov. 2011.

[13] R. W. de Mello, R. Podmore, and S. K. N., "Coherency-Based Dynamic Equivalents: Applications in Transient Stability Studies," Proceedings of PICA Conference, pp. 23-31, 1975.

[14] A. J. Germond and R. Podmore, "Dynamic Aggregation of Generating Unit Models," IEEE Transactions on Power Apparatus and Systems, no. 4, pp. 1060-1069, 1978.

[15] J. Chow, R. Galarza, P. Accari, and W. Price, "Inertial and slow coherency aggregation algorithms for power system dynamic model reduction," IEEE Transactions on Power Systems, vol. 10, no. 2, pp. $680-685$, may 1995

[16] R. A. Date and J. H. Chow, "Aggregation Properties of Linearized Two-Time-Scale Power Networks," IEEE Transactions on Circuits and Systems, vol. 38, no. 7, pp. 720-730, Jul. 1991.

[17] J. H. Chow, "A Nonlinear Model Reduction Formulation for Power System Slow Coherency and Aggregation," in Proceedings of Workshop on Advances in Control and its Applications, ser. Lecture Notes in Control \& Information Sciences, P. I. H.K. Khalil, J.H. Chow, Ed., vol. 208, 1996
[18] — - "New Algorithms for Slow Coherency Aggregation of Large Power Systems," in Systems and Control Theory for Power Systems, ser. IMA Volumes in Mathematics and its Applications, R. J. T. Joe H. Chow, Petar V. Kokotovic, Ed., vol. 64, Institute for Mathematics and its Applications (IMA). Springer-Verlag, 1995, pp. 95-115.

[19] R. Galarza, J. Chow, W. Price, A. Hargrave, and P. Hirsch, "Aggregation of exciter models for constructing power system dynamic equivalents," IEEE Transactions on Power Systems, vol. 13, no. 3, pp. 782 -788, aug 1998.

[20] X. Wang, V. Vittal, and G. Heydt, "Tracing generator coherency indices using the continuation method: A novel approach," Power Systems, IEEE Transactions on, vol. 20, no. 3, pp. 1510 - 1518, aug. 2005.

[21] CORESO, [On-line:] http://www.coreso.eu/, retrieved Nov. 2011

[22] Transmission System Operator Security Cooperation (TCS), [On-line:] http://www.tso-security-cooperation.eu, retrieved Nov. 2011.

[23] M. Brucoli, M. La Scala, N. Pitrone, and M. Trovato, "Dynamic modelling for retaining selected portions of interconnected power networks," IEE Proceedings on Generation, Transmission and Distribution, vol. 135, no. 2, pp. $118-123$, mar 1988.

[24] M. Ibrahim, O. Mostafa, and A. El-Abiad, "Dynamic equivalents using operating data and stochastic modeling," IEEE Transactions on Power Apparatus and Systems, vol. 95, no. 5, pp. 1713 - 1722, sept. 1976.

[25] P. Panciatici, Y. Hassaine, S. Fliscounakis, L. Platbrood, M. OrtegaVazquez, J. Martinez-Ramos, and L. Wehenkel, "Security management under uncertainty: From day-ahead planning to intraday operation," in 2010 iREP Symposium Bulk Power System Dynamics and Control (iREP) - VIII (iREP), aug. 2010, pp. 1 -8.

[26] D. Chaniotis and M. Pai, "Model reduction in power systems using krylov subspace methods," Power Systems, IEEE Transactions on, vol. 20, no. 2, pp. 888-894, 2005.

[27] P. Parrilo, S. Lall, F. Paganini, G. Verghese, B. Lesieutre, and J. Marsden, "Model reduction for analysis of cascading failures in power systems," in American Control Conference, 1999. Proceedings of the 1999, vol. 6. IEEE, 1999, pp. 4208-4212.

[28] D. Enns, "Model reduction with balanced realizations: an error bound and a frequency weighted generalization," in Proceedings of the IEEE Conference on Decision and Control, Las Vegas, Nevada, 1984.

[29] B. Anderson and Y. Liu, "Controller reduction: Concepts and approaches," IEEE Transactions on Automatic Control, vol. 34, pp. 802$812,1989$.

[30] L. Li and F. Paganini, "Structured coprime factor model reduction based on LMIs," Automatica, vol. 41, no. 1, pp. 145-151, Jan. 2005.

[31] A. Vandendorpe and P. Van Dooren, Model Order Reduction: Theory, Research Aspects and Applications, ser. Mathematics in Industry Series. Springer Verlag, 2007, ch. Model reduction of interconnected systems.

[32] H. Sandberg and R. M. Murray, "Model reduction of interconnected linear systems," Optimal Control, Applications and Methods, Special Issue on Directions, Applications, and Methods in Robust Control, vol. $30: 3$, pp. 225-245, 2009.

[33] M. Klein, G. Rogers, and P. Kundur, "A fundamental study of inter-area oscillations in power systems," Power Systems, IEEE Transactions on, vol. 6, no. 3, pp. 914-921, 1991.

[34] T. Glad and L. Ljung, Control theory-Multivariable and nonlinear methods. Taylor and Francis, 2000.

[35] J. Sanchez-Gasca, J. Chow, and R. Galarza, "Reduction of linearized power systems for the study of interarea oscillations," in Control Applications, 1995., Proceedings of the 4th IEEE Conference on, sep 1995, pp. $624-630$.

[36] K. Glover, "All optimal Hankel-norm approximations of linear multivariable systems and their $L_{\infty}$-error bounds," International Journal of Control, vol. 39, pp. 1115-1193, 1984

[37] G. Schelfhout and B. De Moor, "A note on closed-loop balanced truncation," IEEE Transactions on Automatic Control, vol. 41, pp. 14981500, 1996.

[38] P. Wortelboer, "Frequency-weighted balanced reduction of closed-loop mechanical servo-systems: theory and tools," Ph.D. dissertation, TU Delft, Delft University of Technology, 1994-06-20.

[39] F. Milano, "An Open Source Power System Analysis Toolbox," vol. 20, no. 3, pp. 1199-1206, Aug. 2005.

[40] A. C. Antoulas, Approximation of Large-Scale Dynamical Systems. Philadelphia: SIAM, 2005. 